\title{
Dressed (Renormalized) Coordinates in a Nonlinear System
}

\author{
G. Flores-Hidalgo ${ }^{a *}$ and Y. W. Milla ${ }^{b \dagger}$ \\ ${ }^{a}$ Instituto de Física Teorica-IFT/UNESP, \\ Rua Pamplona 145, 01405-900, São Paulo, SP, Brazil \\ ${ }^{b}$ Centro Brasileiro de Pesquisas Fisicas, \\ Rua Dr. Xavier Sigaud 150, 22290-180, Rio de Janeiro, RJ, Brazil
}

August 20, 2018

\begin{abstract}
In previous publications dressed coordinates and dressed states has been introduced in linear systems. Specifically, a system composed by a harmonic oscillator interacting linearly with an infinity set of other oscillators has been treated. In this paper we show how to generalize such dressed coordinates and dressed states to a nonlinear version of this system. Also we clarify some misunderstandings about the concept of dressed coordinates. Indeed now we prefer to call them renormalized coordinates to emphasize the analogy with the renormalized fields in quantum field theory.
\end{abstract}

PACS Number(s): 03.65.Ca, 32.80.Pj

*E-mail: gflores@ift.unesp.br

${ }^{\dagger}$ E-mail: yonym@cbpf.br 


\section{Introduction}

In recent publications it has been introduced the concept of dressed coordinates and dressed states[1, 2, 3, 4], in the context of a harmonic oscillator (the atom) interacting linearly with a massless scalar field. This allowed the construction of dressed states, with the physically correct property of stability of the oscillator ground state in the absence of field quanta (the quantum vacuum). For a review see the next section. Also this formalism allowed a nonperturbative treatment of the oscillator radiation process. When applied to a confined atom (approximated by the oscillator) in a spherical cavity of sufficiently small diameter the method accounts for the experimentally observed inhibition of the decaying processes $[5,6]$.

In Ref. [7] an attempt to construct dressed coordinates and dressed states for a nonlinear system has been done. However the approach used there was more intuitive than formal. The purpose of this paper is to develop a formal method to construct dressed coordinates in nonlinear systems. We will do this by a perturbative expansion in the nonlinear coupling constant. To be specific we consider the model with Hamiltonian given by,

$$
\begin{aligned}
H= & \frac{1}{2}\left(p_{0}^{2}+\omega_{B}^{2} q_{0}^{2}\right)+\frac{1}{2} \sum_{k=1}^{N}\left(p_{k}^{2}+\omega_{k}^{2} q_{k}^{2}-2 c_{k} q_{k} q_{0}\right) \\
& +\sum_{r=0}^{N} \lambda_{r} \mathcal{T}_{\mu \nu \rho \sigma}^{(r)} q_{\mu} q_{\nu} q_{\rho} q_{\sigma}+\sum_{r=0}^{N} \alpha_{r} \mathcal{R}_{\mu \nu \rho \sigma \tau \epsilon}^{(r)} q_{\mu} q_{\nu} q_{\rho} q_{\sigma} q_{\tau} q_{\epsilon},
\end{aligned}
$$

where sums over repeated indices and the limit $N \rightarrow \infty$ are understood. In Eq. (1) the bare frequency of the oscillator, $\omega_{B}$, is related to the physical frequency, $\omega_{0}$, by $[8,9]$,

$$
\omega_{B}^{2}=\omega_{0}^{2}+\sum_{k=1}^{N} \frac{c_{k}^{2}}{\omega_{k}^{2}} .
$$

The coefficients $\mathcal{T}_{\mu \nu \rho \sigma}^{(r)}$ and $\mathcal{R}_{\mu \nu \rho \sigma \tau \epsilon}^{(r)}$ are chosen in such a way that the Hamiltonian given by Eq. (1) is positive defined.

In Ref. [7] the quartic nonlinear model, $\alpha_{r}=0$, has been treated. Here also we will be mainly interested in the quartic nonlinear model to compare with the early treatment. 
The sextic nonlinear interaction will be considered mainly because for some values of $\lambda_{r}$ and $\alpha_{r}$ it is possible to find the exact solution for the ground state and, as explained at the end of section III, this fact will permit an exact construction of the dressed coordinates. Then, this sextic model will allow us to test the validity of the strategy developed to obtain the dressed coordinates in a general nonlinear system.

Along this paper we use natural units $c=\hbar=1$.

\section{Defining dressed (renormalized) coordinates and dressed states}

The purpose od this section is twofold. First, to make this paper self contained we review what was called dressed coordinates and dressed states in the Refs. $[1,2,3]$. Second, we clarify some misunderstandings about the concept of dressed coordinates, as we explain below. Indeed now we prefer to call them renormalized coordinates to emphasize that these coordinates are analogous to the renormalized fields in quantum field theory.

To understand the necessity of introducing dressed coordinates and dressed states let us consider the following problem. Imagine that the oscillator with index zero in Eq. (1) represents an atom and the other oscillators represent the modes of the electromagnetic field. If there are no interaction among them the free Hamiltonian $\left(c_{k}=\lambda_{r}=\alpha_{r}=0\right)$ has the following eigenfunctions,

$$
\begin{aligned}
\psi_{n_{0} n_{1} \ldots n_{N}}(q) & \equiv\left\langle q \mid n_{0}, n_{1}, \ldots, n_{N}\right\rangle \\
& =\prod_{\mu=0}^{N} \psi_{n_{\mu}}\left(q_{\mu}\right)
\end{aligned}
$$

where $|q\rangle=\left|q_{0}, q_{1}, \ldots, q_{N}\right\rangle$ and $\psi_{n_{\mu}}\left(q_{\mu}\right)$ is the eigenfunction of a harmonic oscillator of frequency $\omega_{\mu}$,

$$
\psi_{n_{\mu}}\left(q_{\mu}\right)=\left(\frac{\omega_{\mu}}{\pi}\right)^{1 / 4} \frac{H_{n_{\mu}}\left(\sqrt{\omega_{\mu}} q_{\mu}\right)}{\sqrt{2^{n_{\mu}} n_{\mu} !}} e^{-\frac{1}{2} \omega_{\mu} q_{\mu}^{2}}
$$


The physical meaning of $\psi_{n_{0} n_{1} \ldots n_{N}}(q)$ in this case is clear, it represents the atom in its $n_{0^{-}}$ th excited level and $n_{k}$ photons of frequencies $\omega_{k}$. Now, consider the state of no photons, $\psi_{n_{0} 0 \ldots 0}(q)$ : the excited atom in the quantum vacuum. We know from experience that any excited level of the atom is unstable, that is, the state $\psi_{n_{0} 0 \ldots 0}(q)$ is unstable. The explanation of this fact is that the atom is not isolated from interacting with the quantum electromagnetic field, or in other words it is rendered unstable by interacting with the quantum vacuum. This interaction in our model is given by the linear and nonlinear couplings of $q_{0}$ with $q_{k}$. Obviously, when we take into account these interactions any state of the type $\psi_{n_{0} 0 \ldots 0}(q)$ will be unstable, since these states are not eigenfunctions of the total interacting Hamiltonian. But, there is a problem, the state $\psi_{00 \ldots 0}(q)$, that represents the atom in its ground state and no photons, is also unstable contradicting the experimental fact of the stability of the ground state in the absence of photons. What is wrong in all this? The first thing that cames in our mind is to think that the model given by Eq. (1) is wrong. Certainly, we know that the correct theory to describe this physical system is quantum electrodynamics. On the other hand such a description of this system could be extremely complicated. If we aim to maintain the model as simple as possible and still insist in describing it by the Hamiltonian given in Eq. (1) what we can do in order to take into account the stability of the ground state? The answer lies in the spirit of the renormalization program in quantum fiel theory: the coordinates $q_{\mu}$ that appear in the Hamiltonian given by Eq. (1) are not the physical ones, they are bare coordinates. We introduce renormalized coordinates, $q_{0}^{\prime}$ and $q_{k}^{\prime}$, respectively for the dressed atom and the dressed photons. We define these coordinates as the physically meaningful ones. These renormalized coordinates were called in prededing works as dressed coordinates $[1,2,3]$, for this reason, from now on we will take these denominations as synonymous. In terms of these dressed coordinates we define dressed states as

$$
\begin{aligned}
\psi_{n_{0} n_{1} \ldots n_{N}}\left(q^{\prime}\right) & \equiv\left\langle q^{\prime} \mid n_{0}, n_{1}, \ldots, n_{N}\right\rangle_{d} \\
& =\prod_{\mu=0}^{N} \psi_{n_{\mu}}\left(q_{\mu}^{\prime}\right)
\end{aligned}
$$

where the subscript $d$ means dressed state, $\left|q^{\prime}\right\rangle=\left|q_{0}^{\prime}, q_{1}^{\prime}, \ldots, q_{N}^{\prime}\right\rangle$ and $\psi_{n_{\mu}}\left(q_{\mu}^{\prime}\right)$ is given by 


$$
\psi_{n_{\mu}}\left(q_{\mu}^{\prime}\right)=\left(\frac{\omega_{\mu}}{\pi}\right)^{1 / 4} \frac{H_{n_{\mu}}\left(\sqrt{\omega_{\mu}} q_{\mu}^{\prime}\right)}{\sqrt{2^{n_{\mu}} n_{\mu} !}} e^{-\frac{1}{2} \omega_{\mu}\left(q_{\mu}^{\prime}\right)^{2}} .
$$

The dressed states given by Eq. (5) are defined as the physically measurable states and describe in general, the physical atom in the $n_{0}$-th excited level and $n_{k}$ physical photons of frequency $\omega_{k}$. Obviously, in the limit in which the coupling constants $c_{k}, \lambda_{r}$ and $\alpha_{r}$ vanish the renormalized coordinates $q_{\mu}^{\prime}$ approach the bare coordinates $q_{\mu}$. Now, in order to relate the bare and dressed coordinates we have to use the physical requirement of stability of the dressed ground state. The dressed ground state will be stable only and only if it is defined as the eigenfunction of the interacting Hamiltonian given in Eq. (1). Also the dressed ground state must be the one of minimum energy, that is, it must be defined as being identical (or proportional) to the ground state eigenfunction of the interacting Hamiltonian. From this definition, one can construct the dressed coordinates in terms of the bare ones.

Firstly we explicitly construct the dressed coordinates for the linear model obtained from Eq. (1) by setting $\lambda_{r}=\alpha_{r}=0$,

$$
H_{\text {linear }}=\frac{1}{2}\left(p_{0}^{2}+\omega_{B}^{2} q_{0}^{2}\right)+\frac{1}{2} \sum_{k=1}^{N}\left(p_{k}^{2}+\omega_{k}^{2} q_{k}^{2}-2 c_{k} q_{k} q_{0}\right) .
$$

Although the task of constructing dressed coordinates in linear systems has been done in preceding works, we repeat here the calculation in order to make this paper self contained. In the next section we will consider the nonlinear case. As is well know, the Hamiltonian (7) can be diagonalized by means of the introduction of normal coordinates $Q_{r}$ and momenta $P_{r}$, defined as

$$
q_{\mu}=\sum_{r=0}^{N} t_{\mu}^{r} Q_{r}, \quad p_{\mu}=\sum_{r=0}^{N} t_{\mu}^{r} P_{r}, \quad \mu=(0, k), \quad k=1,2, \ldots, N,
$$

where $\left\{t_{\mu}^{r}\right\}$ is an orthogonal matrix whose elements are given by $[10,11]$

$$
t_{0}^{r}=\left[1+\sum_{k=1}^{N} \frac{c_{k}^{2}}{\left(\omega_{k}^{2}-\Omega_{r}^{2}\right)^{2}}\right]^{-\frac{1}{2}}, \quad t_{k}^{r}=\frac{c_{k}}{\left(\omega_{k}^{2}-\Omega_{r}^{2}\right)} t_{0}^{r} .
$$

In normal coordinates the Hamiltonian (7) reads 


$$
H_{\text {linear }}=\frac{1}{2} \sum_{r=0}^{N}\left(P_{r}^{2}+\Omega_{r}^{2} Q_{r}^{2}\right),
$$

where the $\Omega_{r}$ 's are the normal frequencies, corresponding to the collective modes and given as solutions of $[10,11]$,

$$
\omega_{0}^{2}-\Omega_{r}^{2}=\sum_{k=1}^{N} \frac{c_{k}^{2} \Omega_{r}^{2}}{\omega_{k}^{2}\left(\omega_{k}^{2}-\Omega_{r}^{2}\right)} .
$$

The eigenfunctions of the Hamiltonian given in Eq. (10) are given by

$$
\begin{aligned}
\phi_{n_{0} n_{1} \ldots n_{N}}(Q) & \equiv\left\langle Q \mid n_{0}, n_{1}, \ldots, n_{N}\right\rangle_{c} \\
& =\prod_{r=0}^{N} \phi_{n_{r}}\left(Q_{r}\right),
\end{aligned}
$$

where the subscript $c$ means collective state, $|Q\rangle=\left|Q_{0}, Q_{1}, \ldots, Q_{N}\right\rangle$ and $\phi_{n_{r}}\left(Q_{r}\right)$ are the wave functions corresponding to one dimensional harmonic oscillators of frequencies $\Omega_{r}$,

$$
\phi_{n_{r}}\left(Q_{r}\right)=\left(\frac{\Omega_{r}}{\pi}\right)^{1 / 4} \frac{H_{n_{r}}\left(\sqrt{\Omega_{r}} Q_{r}\right)}{\sqrt{2^{n_{r}} n_{r} !}} e^{-\frac{1}{2} \Omega_{r} Q_{r}^{2}} .
$$

Now, the dressed coordinates are defineed requiring $\psi_{00 \ldots 0}\left(q^{\prime}\right) \propto \phi_{00 \ldots 0}(Q)$, since in this way we guarantee that $\psi_{00 \ldots 0}\left(q^{\prime}\right)$ is the ground state of $H_{\text {linear }}$. Then, from Eqs. (12) and (5), we have

$$
e^{-\frac{1}{2} \sum_{\mu=0}^{N} \omega_{\mu}\left(q_{\mu}^{\prime}\right)^{2}} \propto e^{-\frac{1}{2} \sum_{r=0}^{N} \Omega_{r} Q_{r}^{2}},
$$

from which we obtain

$$
q_{\mu}^{\prime}=\sum_{r=0}^{N} \sqrt{\frac{\Omega_{r}}{\omega_{\mu}}} t_{\mu}^{r} Q_{r},
$$

as can be seen by direct substitution in Eq. (14) and using the orthonormality properties of the $\left\{t_{\mu}^{r}\right\}$ matrix. The above definition guarantees the stability of the dressed ground state, however, since the other dressed states are not energy eigenfunctions, they will not remain stable. For example the first excited dressed state, whose eigenfunction is $\psi_{10 \ldots 0}\left(q^{\prime}\right)$, will decay to the ground state $\psi_{00 \ldots 0}\left(q^{\prime}\right)$. 
We have to remark here that the dressed coordinates here introduced are not simply a change of variables, they are new coordinates in its own right and are introduced by physical consistence requirement of the model. Also we have to stress that our dressed coordinates are not the same as the ones employed in other references, as for example in [12] and references therein, where the authors called dressed coordinates the collective normal ones. Also our dressed states are very different from the ones defined in Refs. $[13,14,15,16]$, where the authors called dressed states the states obtained by diagonalizing a truncated finite matrix representation of the Hamiltonian.

Before leaving this section it will be useful to establish the relation between $\psi_{n_{0} n_{1} \ldots n_{N}}\left(q^{\prime}\right)=\left\langle q^{\prime} \mid n_{0}, n_{1}, \ldots, n_{N}\right\rangle_{d}$ and $\left\langle Q \mid n_{0}, n_{1}, \ldots, n_{N}\right\rangle_{d}$. For this end we write

$$
\begin{aligned}
{ }_{d}\left\langle n_{0}, n_{1}, \ldots, n_{N} \mid m_{0}, m_{1}, \ldots, m_{N}\right\rangle_{d} & =\int d q^{\prime}{ }_{d}\left\langle n_{0}, n_{1}, \ldots, n_{N} \mid q^{\prime}\right\rangle\left\langle q^{\prime} \mid m_{0}, m_{1}, \ldots, m_{N}\right\rangle \\
& =\int d Q\left|\frac{\partial q^{\prime}}{\partial Q}\right|{ }_{d}\left\langle n_{0}, n_{1}, \ldots, n_{N} \mid q^{\prime}\right\rangle\left\langle q^{\prime} \mid m_{0}, m_{1}, \ldots, m_{N}\right\rangle_{d} \\
& =\int d Q{ }_{d}\left\langle n_{0}, n_{1}, \ldots, n_{N} \mid Q\right\rangle\left\langle Q \mid m_{0}, m_{1}, \ldots, m_{N}\right\rangle_{d}, \quad(16)
\end{aligned}
$$

where $d q^{\prime}=\prod_{\mu=0}^{N} d q_{\mu}^{\prime}, d Q=\prod_{r=0}^{N} d Q_{r}$ and $\left|\partial q^{\prime} / \partial Q\right|$ is the Jacobian associated to the transformation $q_{\mu}^{\prime} \rightarrow Q_{r}$. From Eq. (16) we get

$$
\left\langle Q \mid n_{0}, n_{1}, \ldots, n_{N}\right\rangle_{d}=\left|\frac{\partial q^{\prime}}{\partial Q}\right|^{1 / 2}\left\langle q^{\prime} \mid n_{0}, n_{1}, \ldots, n_{N}\right\rangle_{d} .
$$

Taking $n_{0}=n_{1}=\ldots=n_{N}=0$ in Eq. $(17)$ and using $\psi_{00 \ldots 0}\left(q^{\prime}\right) \propto \phi_{00 \ldots 0}(Q)$ we get

$$
|0,0, \ldots, 0\rangle_{d} \propto \int d Q\left|\frac{\partial q^{\prime}}{\partial Q}\right|^{1 / 2}|Q\rangle\langle Q \mid 0,0, \ldots, 0\rangle_{c} .
$$

In the linear case, we easily get, from Eq. (15), $\left|\partial q^{\prime} / \partial Q\right|=\prod_{r, \mu=0}^{N} \sqrt{\Omega_{r} / \omega_{\mu}}$ and using this result in Eq. (18) we obtain

$$
|0,0, \ldots, 0\rangle_{d} \propto|0,0, \ldots, 0\rangle_{c} .
$$

For a nonlinear system, certainly a relation of the type given by Eq. (19) will not hold.

In next section we construct dressed coordinates in the nonlinear model described by the Hamiltonian given in Eq. (1). 


\section{Constructing renormalized coordinates in a non- linear model}

Now we are ready to construct dressed coordinates and dressed states in the nonlinear model given by Eq. (1). For this purpose we have to find, firstly, the eigenfunctions of this Hamiltonian, in particular its ground state eigenfunction.

In order to maintain things as simple as possible and to compare with the the preceding treatment given in Ref. [7], we consider the nonlinear quartic interaction obtained from the model given described in Eq. (1) by setting $\alpha_{r}=0$. Following Ref. [7] we make the simplest choice for the coefficients $\mathcal{T}_{\mu \nu \rho \sigma}^{(r)}$ as

$$
\mathcal{T}_{\mu \nu \rho \sigma}^{(r)}=t_{\mu}^{r} t_{\nu}^{r} t_{\rho}^{r} t_{\sigma}^{r}
$$

Substituting Eqs. (8) and (20) in Eq. (1) we get

$$
H=\frac{1}{2} \sum_{r=0}^{N}\left(P_{r}^{2}+\Omega_{r}^{2} Q_{r}^{2}+2 \lambda_{r} Q_{r}^{4}\right),
$$

that is, we obtain a system of uncoupled quartic anharmonic oscillators. In Eq. (21) we can notice that $\lambda_{r}$ has dimension of $[\text { frequency }]^{3}$. Then we write $\lambda_{r}=\lambda \Omega_{r}^{3}$, where $\lambda$ is a dimensionless coupling constant. The eigenfunctions of the Hamiltonian given by Eq. (21) can be written as

$$
\begin{aligned}
\phi_{n_{0} n_{1} \ldots n_{N}}(Q ; \lambda) & \equiv\left\langle Q \mid n_{0}, n_{1}, \ldots, n_{N} ; \lambda\right\rangle_{c} \\
& =\prod_{r=0}^{N} \phi_{n_{r}}\left(Q_{r} ; \lambda\right)
\end{aligned}
$$

where $\phi_{n_{r}}\left(Q_{r} ; \lambda\right)$ are eigenfunctions of $\left(P_{r}^{2}+\Omega_{r}^{2} Q_{r}^{2}+2 \lambda \Omega_{r}^{3} Q_{r}^{4}\right) / 2$ and can be written formally as (see Appendix)

$$
\phi_{n_{r}}\left(Q_{r} ; \lambda\right)=\left(\frac{\Omega_{r}}{\pi}\right)^{1 / 4}\left[\frac{H_{n_{r}}\left(\sqrt{\Omega_{r}} Q_{r}\right)}{\sqrt{2^{n_{r}} n_{r}} !}+\sum_{l=1}^{\infty} \lambda^{l} G_{n_{r}}^{(l)}\left(\sqrt{\Omega_{r}} Q_{r}\right)\right] e^{-\frac{\Omega_{r}}{2} Q_{r}^{2}}
$$

where $G_{n_{r}}^{(l)}\left(\sqrt{\Omega_{r}} Q_{r}\right)$ are linear combinations of Hermite polynomials. The corresponding eigenvalues for the Hamiltonian given in Eq. (21) are given by, 


$$
E_{n_{0} n_{1} \ldots n_{N}}(\lambda)=\sum_{r=0}^{N} E_{n_{r}}(\lambda),
$$

where $E_{n_{r}}(\lambda)$ are the eigenvalues corresponding to the eigenstates given in Eq. (23),

$$
E_{n_{r}}(\lambda)=\left(n_{r}+\frac{1}{2}\right) \Omega_{r}+\sum_{l=1}^{\infty} \lambda^{l} E_{n_{r}}^{(l)},
$$

with the $E_{n_{r}}^{(l)}$ obtained by using standard perturbation theory (see Appendix).

Taking $n_{0}=n_{1}=\ldots=n_{N}=0$ in Eq. (22) we get for the ground state eigenfunction of the total system,

$$
\phi_{00 \ldots 0}(Q ; \lambda)=\prod_{r=0}^{N}\left(\frac{\Omega_{r}}{\pi}\right)^{1 / 4}\left[1+\sum_{l=1}^{\infty} \lambda^{l} G_{0}^{(l)}\left(\sqrt{\Omega_{r}} Q_{r}\right)\right] e^{-\frac{\Omega_{r}}{2} Q_{r}^{2}} .
$$

To properly define (see comments below) the dressed coordinates it is convenient to write the above equation as,

$$
\begin{aligned}
\phi_{00 \ldots 0}(Q ; \lambda)= & \prod_{r=0}^{N}\left(\frac{\Omega_{r}}{\pi}\right)^{1 / 4}\left[1+\sum_{l=1}^{\infty} \lambda^{l} G_{0}^{(l)}(0)+\sum_{l=1}^{\infty} \lambda^{l}\left(G_{0}^{(l)}\left(\sqrt{\Omega_{r}} Q_{r}\right)-G_{0}^{(l)}(0)\right)\right] e^{-\frac{\Omega_{r}}{2} Q_{r}^{2}} \\
\propto & \prod_{r=0}^{N}\left[1+\sum_{n=0}^{\infty}(-1)^{n} \sum_{l_{0} l_{1} \ldots l_{n}=1}^{\infty} \lambda^{l_{0}+l_{1}+\ldots+l_{n}}\right. \\
& \left.\quad \times\left(G_{0}^{\left(l_{0}\right)}\left(\sqrt{\Omega_{r}} Q_{r}\right)-G_{0}^{\left(l_{0}\right)}(0)\right) G_{0}^{\left(l_{1}\right)}(0) \ldots G_{0}^{\left(l_{n}\right)}(0)\right] e^{-\frac{\Omega_{r}}{2} Q_{r}^{2}}, \quad(27)
\end{aligned}
$$

where in the second line we factored the term $1+\sum_{l=1}^{\infty} \lambda^{l} G_{0}^{(l)}(0)$ and used $(1+x)^{-1}=$ $\sum_{n=0}^{\infty}(-1)^{n} x^{n}$

The physically measurable states, the dressed states, are defined by Eqs. (5) and (6). Hence, the dressed coordinates $q_{\mu}^{\prime}$ will be defined in such a way that the dressed ground state equals (or is proportional) to the ground state of the nonlinear interacting Hamiltonian given in Eq. (27). That is, we define the dressed coordinates imposing the condition $\psi_{00 \ldots 0}\left(q^{\prime}\right) \propto \phi_{00 \ldots 0}(Q ; \lambda)$ which by using Eqs. (5), (6) and $(27)$ can be written as

$$
\begin{aligned}
e^{-\frac{1}{2} \sum_{\mu=0}^{N} \omega_{\mu}\left(q_{\mu}^{\prime}\right)^{2}}=\prod_{r=0}^{N}[1+ & \sum_{n=0}^{\infty}(-1)^{n} \sum_{l_{0} l_{1} \ldots l_{n}=1}^{\infty} \lambda^{l_{0}+l_{1}+\ldots+l_{n}} \\
& \left.\times\left(G_{0}^{\left(l_{0}\right)}\left(\sqrt{\Omega_{r}} Q_{r}\right)-G_{0}^{\left(l_{0}\right)}(0)\right) G_{0}^{\left(l_{1}\right)}(0) \ldots G_{0}^{\left(l_{n}\right)}(0)\right] e^{-\frac{\Omega_{r}}{2} Q_{r}^{2}} .
\end{aligned}
$$


Now, we write a perturbative expansion in $\lambda$ for $q_{\mu}^{\prime}$,

$$
q_{\mu}^{\prime}=\sum_{r=0}^{N} \sqrt{\frac{\Omega_{r}}{\omega_{\mu}}} t_{\mu}^{r}\left[Q_{r}+\frac{1}{\sqrt{\Omega_{r}}} \sum_{l=1}^{\infty} \lambda^{l} F_{r}^{(l)}\left(\sqrt{\Omega_{r}} Q_{r}\right)\right] .
$$

Replacing Eq. (29) in Eq. (28) and using the the orthonormality of the matrix $\left\{t_{\mu}^{r}\right\}$ we get

$$
\begin{aligned}
& \exp \left[-\sum_{l=1}^{\infty} \lambda^{l} \sqrt{\Omega_{r}} Q_{r} F_{r}^{(l)}\left(\sqrt{\Omega_{r}} Q_{r}\right)-\frac{1}{2} \sum_{l, m=1}^{\infty} \lambda^{l+m} F_{r}^{(l)}\left(\sqrt{\Omega_{r}} Q_{r}\right) F_{r}^{(m)}\left(\sqrt{\Omega_{r}} Q_{r}\right)\right] \\
= & 1+\sum_{n=0}^{\infty}(-1)^{n} \sum_{l_{0} l_{1} \ldots l_{n}=1}^{\infty} \lambda^{l_{0}+l_{1}+\ldots+l_{n}}\left(G_{0}^{\left(l_{0}\right)}\left(\sqrt{\Omega_{r}} Q_{r}\right)-G_{0}^{\left(l_{0}\right)}(0)\right) G_{0}^{\left(l_{1}\right)}(0) \ldots G_{0}^{\left(l_{n}\right)}(0) .
\end{aligned}
$$

Expanding the exponential in the left hand side of Eq. (30) and identifying equal powers of $\lambda$ we can obtain all the $F_{r}^{(l)}\left(\sqrt{\Omega_{r}} Q\right)$. The general expression is very complicated, here we only write the first three terms:

$$
\begin{gathered}
F_{r}^{(1)}\left(\xi_{r}\right)=-\frac{1}{\xi_{r}}\left(G_{0}^{(1)}\left(\xi_{r}\right)-G_{0}^{(1)}(0)\right) \\
F_{r}^{(2)}\left(\xi_{r}\right)=-\frac{1}{\xi_{r}}\left[G_{0}^{(2)}\left(\xi_{r}\right)-G_{0}^{(2)}(0)+G_{0}^{(1)}(0) \xi_{r} F_{r}^{(1)}\left(\xi_{r}\right)+\frac{1}{2}\left(1-\xi_{r}^{2}\right)\left(F_{r}^{(1)}\left(\xi_{r}\right)\right)^{2}\right]
\end{gathered}
$$

and

$$
\begin{aligned}
F_{r}^{(3)}\left(\xi_{r}\right)=-\frac{1}{\xi_{r}}[ & G_{0}^{(3)}\left(\xi_{r}\right)-G_{0}^{(3)}(0)-\left(G_{0}^{(2)}\left(\xi_{r}\right)-G_{0}^{(2)}(0)\right) G_{0}^{(1)}(0) \\
& +\xi_{r} F_{r}^{(1)}\left(\xi_{r}\right)\left(G_{0}^{(2)}(0)-\left(G_{0}^{(1)}(0)\right)^{2}\right)+\frac{\xi_{r}}{3 !}\left(\xi_{r}^{2}-3\right)\left(F_{r}^{(1)}\left(\xi_{r}\right)\right)^{3} \\
& \left.+\left(1-\xi_{r}^{2}\right) F_{r}^{(1)}\left(\xi_{r}\right) F_{r}^{(2)}\left(\xi_{r}\right)\right]
\end{aligned}
$$

where $\xi_{r}=\sqrt{\Omega_{r}} Q_{r}$. From the Appendix, using Eqs. (68-71) in Eqs. (31) and (32) we get respectively,

$$
F_{r}^{(1)}\left(\xi_{r}\right)=\frac{1}{4}\left(3 \xi_{r}+\xi_{r}^{3}\right)
$$

and 


$$
F_{r}^{(2)}\left(\xi_{r}\right)=-\frac{1}{16}\left(\frac{93}{2} \xi_{r}+14 \xi_{r}^{3}+\frac{11}{6} \xi_{r}^{5}\right) .
$$

Replacing above equations in Eq. (29) we obtain at order $\lambda^{2}$,

$$
\xi_{\mu}^{\prime}=\sum_{r=0}^{N} t_{\mu}^{r}\left[\xi_{r}+\frac{\lambda}{4}\left(3 \xi_{r}+\xi_{r}^{3}\right)-\frac{\lambda^{2}}{16}\left(\frac{93}{2} \xi_{r}+14 \xi_{r}^{3}+\frac{11}{6} \xi_{r}^{5}\right)+\mathcal{O}\left(\lambda^{3}\right)\right],
$$

where we have introduced the dimensionless dressed coordinate $\xi_{\mu}^{\prime}=\sqrt{\omega_{\mu}} q_{\mu}^{\prime}$.

Before leaving this section we would like to comment why we factored the term $1+\sum_{l=1}^{\infty} \lambda^{l} G_{0}^{(l)}(0)$ in Eq. (27). Notice that we define the dressed coordinates $q_{\mu}^{\prime}$ by means of the proportionality $\psi_{00 \ldots 0}\left(q^{\prime}\right) \propto \phi_{00 \ldots 0}(Q ; \lambda)$. To promote this proportionality into an equality we have to take care in defining a well behaved transformation between dressed and collective coordinates, for example it would be undesirable any singular transformation. To see how the above undesirable situation occurs, define the dressed coordinates through Eq. (26) without the factorization of the term $1+\sum_{l=1}^{\infty} \lambda^{l} G_{0}^{(l)}(0)$. It is easy to show that in such a case the transformation between $q_{\mu}^{\prime}$ and $Q_{r}$ is singular. For example we obtain for $F_{r}^{1}\left(\xi_{r}\right)$ and $F_{r}^{2}\left(\xi_{r}\right)$

$$
\begin{gathered}
F_{r}^{(1)}\left(\xi_{r}\right)=-\frac{1}{\xi_{r}} G_{0}^{(1)}\left(\xi_{r}\right) \\
F_{r}^{(2)}\left(\xi_{r}\right)=-\frac{1}{\xi_{r}}\left[G_{0}^{(2)}\left(\xi_{r}\right)+\frac{1}{2}\left(1-\xi_{r}^{2}\right)\left(F_{r}^{(1)}\left(\xi_{r}\right)\right)^{2}\right]
\end{gathered}
$$

Since $G_{0}^{(1)}\left(\xi_{r}\right)$ and $G_{0}^{(2)}\left(\xi_{r}\right)$ are not homogeneus functions of $\xi_{r}$ [see Appendix, Eqs. (68) and (69)] then Eqs. (37) and (38) are singular in $\xi_{r}=0$. Consequently, the dressed coordinates defined through this prescription are not well defined. To understand what is happening and how to remedy this problem, note that this singularity means that $\xi_{r} F_{r}^{(l)}\left(\xi_{r}\right)$ is not homegeneus in $\xi_{r}$. But the effect of this nonhomogeneous term on the wave function [that contains terms of the type $e^{-\lambda^{l} \xi_{r} F_{r}^{(l)}\left(\xi_{r}\right)}$, see Eq. (30)] is just equal to a factorization term. Then to remedy the situation we have to make a convenient factorization in $\psi_{00 . .0}\left(q^{\prime}\right)$ or, equivalently, in $\phi_{00 . .0}(Q)$ before promoting the proportionality into an equality. That our choice, the factorization of $1+\sum_{l=1}^{\infty} \lambda^{l} G_{0}^{(l)}(0)$ in Eq. (27), is the correct one is supported by the fact that we obtain well behaved dressed coordinates. To further support our choice, we consider a system in which we can solve exactly for 
the ground state, allowing us to obtain exact dressed coordinates. Comparing these exact dressed coordinates and the perturbative ones we get the same answer. The model in mention is the one whose Hamiltonian is given by Eq. (1) with coupling constants defined as

$$
\lambda_{r} \mathcal{T}_{\mu \nu \rho \sigma}^{(r)}=\frac{\lambda \Omega_{r}^{3}}{(1-3 \lambda)^{3 / 2}} t_{\mu}^{r} t_{\nu}^{r} t_{\rho}^{r} t_{\sigma}^{r}, \quad \alpha_{r} \mathcal{R}_{\mu \nu \rho \sigma \tau \epsilon}^{(r)}=\frac{\lambda^{2} \Omega_{r}^{4}}{2(1-3 \lambda)^{2}} t_{\mu}^{r} t_{\nu}^{r} t_{\rho}^{r} t_{\sigma}^{r} t_{\tau}^{r} t_{\epsilon}^{r} .
$$

Using the above expression in Eq. (1) we get a system of uncoupled sextic anharmonic oscillators,

$$
H=\frac{1}{2} \sum_{r=0}^{N}\left(P_{r}^{2}+\Omega_{r}^{2} Q_{r}^{2}+\frac{2 \lambda \Omega_{r}^{3}}{(1-3 \lambda)^{3 / 2}} Q_{r}^{4}+\frac{\lambda^{2} \Omega_{r}^{4}}{(1-3 \lambda)^{2}} Q_{r}^{6}\right) .
$$

By direct substitution it is easy to show that the above Hamiltonian have as ground state eigenfunction the following solution [17]

$$
\phi(Q ; \lambda)=\mathcal{N} e^{-\sum_{r=0}^{N}\left(\beta_{r} Q_{r}^{2}+\lambda \beta_{r}^{2} Q_{r}^{4}\right)},
$$

where $\mathcal{N}$ is a normalization constant,

$$
\beta_{r}=\frac{\Omega_{r}}{2 \sqrt{1-3 \lambda}}
$$

and the corresponding ground state energy is given by

$$
E(\lambda)=\sum_{r=0}^{N} \beta_{r} .
$$

Now, the dressed coordinates can be defined by

$$
e^{-\sum_{\mu=0}^{N} \omega_{\mu}\left(q_{\mu}^{\prime}\right)^{2}}=e^{-\sum_{r=0}^{N}\left(\beta_{r} Q_{r}^{2}+\lambda \beta_{r}^{2} Q_{r}^{4}\right)},
$$

from which we obtain

$$
\xi_{\mu}^{\prime}=\sum_{r=0}^{N} t_{\mu}^{r} \xi_{r}\left(\frac{1}{\sqrt{1-3 \lambda}}+\frac{\lambda \xi_{r}^{2}}{2(1-3 \lambda)}\right)^{1 / 2} .
$$

Note that at order $\lambda$ both the quartic and sextic anharmonic Hamiltonians, given respectively by Eqs. (21) and (40), are equivalent. Then, if our strategy to define perturbatively 
the dressed coordinates is correct, at order $\lambda$ Eq. (36) must be identical to Eq. (45). Expanding Eq. (45) at order $\lambda$ we can see that it is indeed the case. Then we conclude that our strategy for defining perturbatively the dressed coordinates is the correct one.

\section{The decay process of the first excited state}

In Ref. [7] the probability of the particle oscillator to remain in the first excited state has been computed at first order for the nonlinear quartic interaction. However, as we have already mentioned, the approach used there was more intuitive than formal. In order to see in what extent such calculation is correct, in this section we compute the same quantity by using the formalism presented in the last section. To maintain the reasoning as general as possible we present the steps necessary to compute the probability amplitude associated with the most general transition,

$$
\mathcal{A}_{n_{0} n_{1} \ldots n_{N}}^{m_{0} m_{1} \ldots m_{N}}(t)={ }_{d}\left\langle m_{0}, m_{1}, \ldots, m_{N}\left|e^{-i H t}\right| n_{0}, n_{1}, \ldots, n_{N}\right\rangle_{d}
$$

that is, we prepare our system initially at time $t=0$ in the dressed state $\left|n_{0}, n_{1}, \ldots, n_{N}\right\rangle_{d}$, then we ask what is the probability amplitude of finding, in a measurement performed at time $t$, the dressed state $\left|m_{0}, m_{1}, \ldots, m_{N}\right\rangle_{d}$. Introducing a complete set of eigenstates of the total Hamiltonian $H$, given by Eq. (22), in Eq. (46) we find

$$
\begin{aligned}
\mathcal{A}_{n_{0} n_{1} \ldots n_{N}}^{m_{0} m_{1} \ldots m_{N}}(t) & =\sum_{l_{0} l_{1} \ldots l_{N}=0}^{\infty}{ }_{d}\left\langle m_{0}, m_{1}, \ldots, m_{N}\left|e^{-i H t}\right| l_{0}, l_{1}, \ldots, l_{N} ; \lambda\right\rangle_{c c}\left\langle l_{0}, l_{1}, \ldots, l_{N} ; \lambda \mid n_{0}, n_{1}, \ldots, n_{N}\right\rangle_{d} \\
& =\sum_{l_{0} l_{1} \ldots l_{N}=0}^{\infty} T_{n_{0} n_{1} \ldots n_{N}}^{l_{0} l_{1} \ldots l_{N}}(\lambda) T_{m_{0} m_{1} \ldots m_{N}}^{l_{0} l_{1} \ldots l_{N}}(\lambda) e^{-i t E_{l_{0} l_{1} \ldots l_{N}}(\lambda)}
\end{aligned}
$$

with

$$
\begin{aligned}
T_{n_{0} n_{1} \ldots n_{N}}^{l_{0} l_{1} \ldots l_{N}}(\lambda) & =\int d Q{ }_{c}\left\langle l_{0}, l_{1}, \ldots, l_{N} ; \lambda \mid Q\right\rangle\left\langle Q \mid n_{0}, n_{1}, \ldots, n_{N}\right\rangle_{d} \\
& =\int d Q\left|\frac{\partial q^{\prime}}{\partial Q}\right|^{1 / 2} \phi_{l_{0} l_{1} \ldots l_{N}}(Q ; \lambda) \psi_{n_{0} n_{1} \ldots n_{N}}\left(q^{\prime}\right)
\end{aligned}
$$

where in the second line we have used Eq. (17). 
From Eq. (29) we get easily the Jacobian $\left|\partial q^{\prime} / \partial Q\right|$,

$$
\left|\frac{\partial q^{\prime}}{\partial Q}\right|=\prod_{r, \mu=0}^{N}\left|\sqrt{\frac{\Omega_{r}}{\omega_{\mu}}}\left(1+\frac{1}{\sqrt{\Omega_{r}}} \sum_{l=1}^{\infty} \lambda^{l} \frac{\partial}{\partial Q_{r}} F_{r}^{(l)}\left(\sqrt{\Omega_{r}} Q_{r}\right)\right)\right| .
$$

Now we evaluate, at first order in $\lambda$, the probability amplitude to the particle oscillator remain at time $t$ in the first excited state if it has been prepared in that state at time $t=0$. This quantity is obtained taking $n_{0}=m_{0}=1$ and $n_{k}=m_{k}=0$ in Eq. (47). Notice that to compute $\mathcal{A}_{10 \ldots 0}^{10 \ldots 0}(t)$ at first order in $\lambda$ we need to find $T_{10 \ldots 0}^{l_{0} l_{1} \ldots l_{N}}(\lambda)$, defined in Eq. (48), at order $\lambda$. Replacing Eq. (34) in Eq. (49) we get,

$$
\left|\frac{\partial q^{\prime}}{\partial Q}\right|=\left(\prod_{\mu, r=0}^{N} \frac{\Omega_{r}}{\omega_{\mu}}\right)^{1 / 4}\left[1+\frac{3 \lambda}{32} \sum_{s=0}^{N}\left(6 H_{0}\left(\sqrt{\Omega_{s}} Q_{s}\right)+H_{2}\left(\sqrt{\Omega_{s}} Q_{s}\right)\right]+\mathcal{O}\left(\lambda^{2}\right) .\right.
$$

At order $\lambda$, from Eq. (22), we have for $\phi_{l_{0} l_{1} \ldots l_{N}}(Q, \lambda)$,

$$
\phi_{l_{0} l_{1} \ldots l_{N}}(Q, \lambda)=\prod_{r=0}^{N} \phi_{l_{r}}\left(Q_{r}\right)+\lambda \sum_{r=0}^{N}\left[\left(\frac{\Omega_{r}}{\pi}\right)^{1 / 4} G_{l_{r}}^{(1)}\left(\sqrt{\Omega_{r}} Q_{r}\right) e^{-\frac{\Omega_{r}}{2} Q_{r}^{2}} \prod_{s \neq r} \phi_{l_{s}}\left(Q_{s}\right)\right]+\mathcal{O}\left(\lambda^{2}\right)
$$

where the $\phi_{l_{r}}\left(Q_{r}\right)$ are given by Eq. (13),

$$
\begin{aligned}
G_{l_{r}}^{(1)}\left(\sqrt{\Omega_{r}} Q_{r}\right)= & a_{l_{r}} H_{l_{r}-4}\left(\sqrt{\Omega_{r}} Q_{r}\right)+b_{l_{r}} H_{l_{r}-2}\left(\sqrt{\Omega_{r}} Q_{r}\right)+c_{l_{r}} H_{l_{r}+2}\left(\sqrt{\Omega_{r}} Q_{r}\right) \\
& +d_{l_{r}} H_{l_{r}+4}\left(\sqrt{\Omega_{r}} Q_{r}\right)
\end{aligned}
$$

and $a_{l_{r}}, b_{l_{r}}, c_{l_{r}}$ and $d_{l_{r}}$ are given in Appendix, Eq. (70). Using Eq. (28) and Eq. (29) we have for $\psi_{10 \ldots 0}\left(q^{\prime}\right)$,

$$
\begin{aligned}
\psi_{10 \ldots 0}\left(q^{\prime}\right)= & \left(\prod_{\mu=0}^{N} \frac{\omega_{\mu}}{\pi}\right)^{1 / 4} \frac{H_{1}\left(\sqrt{\omega_{0}} q_{0}^{\prime}\right)}{\sqrt{2}} e^{-\frac{1}{2} \sum_{\mu=0}^{N} \omega_{\mu}\left(q_{\mu}^{\prime}\right)^{2}} \\
= & \left(\prod_{\mu=0}^{N} \frac{\omega_{\mu}}{\pi}\right)^{1 / 4} \sum_{r=0}^{N} \frac{t_{0}^{r}}{\sqrt{2}}\left[H_{1}\left(\sqrt{\Omega_{r}} Q_{r}\right)+2 \lambda F_{r}^{(1)}\left(\sqrt{\Omega_{r}} Q_{r}\right)\right. \\
& \left.-\lambda H_{1}\left(\sqrt{\Omega_{r}} Q_{r}\right) \sum_{s=0}^{N} \sqrt{\Omega_{s}} Q_{s} F_{s}^{(1)}\left(\sqrt{\Omega_{s}} Q_{s}\right)\right] e^{-\frac{1}{2} \sum_{u=0}^{N} \Omega_{u} Q_{u}^{2}}+\mathcal{O}\left(\lambda^{2}\right)
\end{aligned}
$$


Replacing Eqs. (50), (51) and (53) in Eq. (48) we obtain after a long, but straightforward, calculation

$$
\begin{aligned}
T_{10 \ldots 0}^{l_{0} l_{1} \ldots l_{N}}(\lambda)= & \sum_{r=0}^{N} t_{0}^{r} \delta_{l_{r} 1} \prod_{s \neq r} \delta_{l_{s} 0}+9 \frac{\sqrt{6}}{16} \lambda \sum_{r=0}^{N} t_{0}^{r} \delta_{l_{r} 3} \prod_{s \neq r} \delta_{l_{s} 0} \\
& +3 \frac{\sqrt{2}}{16} \lambda \sum_{r \neq s} t_{0}^{r} \delta_{l_{r} 1} \delta_{l_{s} 2} \prod_{u \neq r, s} \delta_{l_{u} 0}+\mathcal{O}\left(\lambda^{2}\right) .
\end{aligned}
$$

Replacing Eq. (54) in Eq. (47) and using from the Appendix $E_{1_{r}}(\lambda) \approx \frac{3}{2} \Omega_{r}+\frac{15}{4} \lambda \Omega_{r}$ we obtain for $\mathcal{A}_{10 \ldots 0}^{10 \ldots 0}(t)$, which we denote as $f_{00}(t ; \lambda)$,

$$
f_{00}(t ; \lambda)=e^{-\frac{i t}{2} \sum_{r=0}^{N} \Omega_{r}} \sum_{r=0}^{N}\left(t_{0}^{r}\right)^{2}\left(1-\frac{15}{4} i \lambda t \Omega_{r}\right) e^{-i t \Omega_{r}}+\mathcal{O}\left(\lambda^{2}\right) .
$$

From the above equation we get the probability to the particle oscillator remain in the first excited level,

$$
\left|f_{00}(t ; \lambda)\right|^{2}=\left|f_{00}(t)\right|^{2}+\frac{15 \lambda t}{4} \frac{\partial}{\partial t}\left|f_{00}(t)\right|^{2}+\mathcal{O}\left(\lambda^{2}\right)
$$

where

$$
f_{00}(t)=\sum_{r=0}^{N}\left(t_{0}^{r}\right)^{2} e^{-i \Omega_{r} t} .
$$

Equation (56) is the same as the one obtained in Ref. [7]. We obtained the same result because at order $\lambda$ the square of $T_{10 \ldots 0}^{l_{0} l_{1} \ldots l_{N}}$ is given only by the square of the first term in Eq. (54), that does not depend on $\lambda$. Then, the effects of the nonlinearities, at this order, cames only from the corrections to the energies, as was assumed in Ref. [7].

\section{Conclusions}

In this paper, after clarifying what we understand by dressed coordinates and dressed states, we have developed a formal method to construct perturbatively dressed coordinates in nonlinear systems. Although we restricted our calculations to a very special quartic interacting term, we have pointed out the necessity of factoring a term in order to avoid 
artificial singularities which otherwise would appear if we do not make such factorization. That this factorization is the correct one has been checked by using an exactly solvable sextic interacting model. Then, in more general nonlinear systems, one can follow the same procedure to construct the dressed coordinates.

At the end of section II we remarked that for nonlinear systems, in the number representation, the dressed ground state is not equivalent to the ground state of the total system, see Eq. (18). This fact must not be seen as in contradiction with our definition of dressed coordinates, since we have defined them by requiring the equivalence of the dressed ground state in dressed coordinates representation and the ground state of the system in normal coordinates representation. We can understand the mentioned non equivalence, by noting that although the dressed ground state is an eigenstate of the dressed number operators (associated with the dressed coordinates) the ground state of the system, in general, is not an eigenstate of the collective (normal) number operators. For example, in the quartic nonlinear case one can easily verify that the ground state (and also, the other eigenstates) is not an eigenstate of the collective number operators, but a linear superposition of eigenstates of these operators [see Appendix, Eq. (62)].

Finally, we considered the computation of the probability of the particle oscillator to remain excited in its first excited level, and showed that the result coincides with the result obtained in Ref. [7]. Then, one of the conclusions of Ref. [7] remains: the effect of the nonlinear quartic terms is the enhancement of the decay of the particle oscillator from its first excited level to the ground state. This fact, can be easily seen from Eq. (56) by noticing that $\left|f_{00}(t)\right|^{2}$ (the probability to the particle oscillator remain in the first excited state in the absence of nonlinear interactions) in free space, is a decreasing (almost exponentially) function of time.

\section{Acknowledgements}

We acknowledge A. P. C. Malbouisson (CBPF) for reading the manuscript. GFH is supported by FAPESP, grant 02/09951-3 and YWM is supported by a grant from CNPq (Conselho Nacional de Desenvolvimento Cientifico e Tecnológico). 


\section{Appendix: The perturbed eigenfunctions and eigen-}

\section{values}

It is easy to see that the eigenfunctions of the quartic anharmonic oscillator can be written formally as those given in Eq. (23). We have to notice only that any wave function can be expanded in the basis $\phi_{n}(Q)$ (we omit here the index $r$ ), given by the eigenvalues of the linear part of the Hamiltonian. And since $\phi_{n}(Q)$ are given by $\exp \left(-\Omega Q^{2} / 2\right)$ times a Hermite polynomial of degree $n$, we see that an expression of the type given in Eq. (23) follows. In what follows we compute $G_{n}^{(1)}(\sqrt{\Omega} Q)$ and $G_{n}^{(2)}(\sqrt{\Omega} Q)$ by using standard perturbation theory.

At second order in standard perturbation theory the eigenfunctions and eigenvalues of a Hamiltonian $\hat{H}=\hat{H}_{0}+\lambda \hat{V}$ are given respectively by,

$|n, \lambda\rangle=|n\rangle+\lambda \sum_{k \neq n} \frac{V_{k n}|k\rangle}{E_{n}-E_{k}}+\lambda^{2}\left(\sum_{k, l \neq n} \frac{V_{k l} V_{l n}|k\rangle}{\left(E_{n}-E_{k}\right)\left(E_{n}-E_{l}\right)}-V_{n n} \sum_{k \neq n} \frac{V_{k n}|k\rangle}{\left(E_{n}-E_{k}\right)^{2}}\right)+\mathcal{O}\left(\lambda^{3}\right)$

and

$$
E_{n}(\lambda)=E_{n}+\lambda V_{n n}+\lambda^{2} \sum_{k \neq n} \frac{\left|V_{n k}\right|^{2}}{E_{n}-E_{k}}+\mathcal{O}\left(\lambda^{3}\right)
$$

where

$$
V_{k n}=\langle k|\hat{V}| n\rangle
$$

and $|n\rangle$ and $E_{n}$ are respectively eigenfunctions and eigenvalues of the unperturbed Hamiltonian $\hat{H}_{0}$.

For the anharmonic oscillator with $\hat{V}=\Omega^{3} \hat{Q}^{4}$ we obtain easily

$$
\begin{aligned}
V_{k n}=\frac{\Omega}{4}[ & \sqrt{k_{4}} \delta_{k, n-4}+2(2 n-1) \sqrt{k_{2}} \delta_{k, n-2}+3\left(2 n^{2}+2 n+1\right) \delta_{k, n} \\
& \left.+2(2 n+3) \sqrt{n_{2}} \delta_{k, n+2}+\sqrt{n_{4}} \delta_{k, n+4}\right]
\end{aligned}
$$


where $k_{n}=(k+1)(k+2) \ldots(k+n)$. Replacing Eq. (61) in Eqs. (58) and (59) we obtain respectively,

$$
\begin{aligned}
|n, \lambda\rangle=|n\rangle & +\lambda\left(a_{n}^{\prime}|n-4\rangle+b_{n}^{\prime}|n-2\rangle+c_{n}^{\prime}|n+2\rangle+d_{n}^{\prime}|n+4\rangle\right) \\
& +\lambda^{2}\left(e_{n}^{\prime}|n-8\rangle+f_{n}^{\prime}|n-6\rangle+g_{n}^{\prime}|n-4\rangle+h_{n}^{\prime}|n-2\rangle\right. \\
& \left.\quad+t_{n}^{\prime}|n+2\rangle+u_{n}^{\prime}|n+4\rangle+v_{n}^{\prime}|n+6\rangle+w_{n}^{\prime}|n+8\rangle\right)+\mathcal{O}\left(\lambda^{3}\right)
\end{aligned}
$$

and

$$
E_{n}(\lambda)=\left(n+\frac{1}{2}\right) \Omega+\lambda E_{n}^{(1)}+\lambda^{2} E_{n}^{(2)}+\mathcal{O}\left(\lambda^{3}\right)
$$

where

$$
\begin{gathered}
a_{n}^{\prime}=\frac{1}{16} \sqrt{(n-4)_{4}}, \quad b_{n}^{\prime}=\frac{(2 n-1)}{4} \sqrt{(n-2)_{2}}, \\
c_{n}^{\prime}=-\frac{(2 n+3)}{4} \sqrt{n_{2}}, \quad d_{n}^{\prime}=-\frac{1}{16} \sqrt{n_{4}} ; \\
e_{n}^{\prime}=\frac{1}{512} \sqrt{(n-8)_{8}}, \quad f_{n}^{\prime}=\frac{(6 n-11)}{192} \sqrt{(n-6)_{6}}, \\
g_{n}^{\prime}=\frac{1}{16}\left(2 n^{2}-9 n+7\right) \sqrt{(n-4)_{4}}, \quad h_{n}^{\prime}=-\frac{1}{64}\left(2 n^{3}+93 n^{2}-107 n+66\right) \sqrt{(n-2)_{2}} \\
t_{n}^{\prime}=-\frac{1}{64}\left(2 n^{3}-123 n^{2}-359 n-300\right) \sqrt{n_{2}}, \quad u_{n}^{\prime}=\frac{1}{16}\left(2 n^{2}+13 n+18\right) \sqrt{n_{4}}, \\
v_{n}^{\prime}=\frac{(6 n+17)}{192} \sqrt{n_{6}}, \quad w_{n}^{\prime}=\frac{1}{512} \sqrt{n_{8}} ; \\
E_{n}^{(1)}=\frac{3}{4}\left(2 n^{2}+2 n+1\right) \Omega
\end{gathered}
$$

and

$$
E_{n}^{(2)}=-\frac{1}{8}\left(34 n^{3}+51 n^{2}+59 n+21\right) \Omega .
$$

Writing Eq. (62), in coordinate representation, in the form given in Eq. (23) we get for $G_{n}^{(1)}(\sqrt{\Omega} Q)$ and $G_{n}^{(2)}(\sqrt{\Omega} Q)$ respectively, 


$$
G_{n}^{(1)}(\xi)=a_{n} H_{n-4}(\xi)+b_{n} H_{n-2}(\xi)+c_{n} H_{n+2}(\xi)+d_{n} H_{n+4}(\xi)
$$

and

$$
\begin{aligned}
G_{n}^{(2)}(\xi)= & e_{n} H_{n-8}(\xi)+f_{n} H_{n-6}(\xi)+g_{n} H_{n-4}(\xi)+h_{n} H_{n-2}(\xi) \\
& +t_{n} H_{n+2}(\xi)+u_{n} H_{n+4}(\xi)+v_{n} H_{n+6}(\xi)+w_{n} H_{n+8}(\xi)
\end{aligned}
$$

where $\xi=\sqrt{\Omega} Q$,

$$
\begin{aligned}
& a_{n}=\frac{a_{n}^{\prime}}{\sqrt{2^{n-4}(n-4) !}}, \quad b_{n}=\frac{b_{n}^{\prime}}{\sqrt{2^{n-2}(n-2) !}}, \\
& c_{n}=\frac{c_{n}^{\prime}}{\sqrt{2^{n+2}(n+2) !}}, \quad d_{n}=\frac{d_{n}^{\prime}}{\sqrt{2^{n+4}(n+4) !}}
\end{aligned}
$$

and

$$
\begin{array}{ll}
e_{n}=\frac{e_{n}^{\prime}}{\sqrt{2^{n-8}(n-8) !}}, \quad f_{n}=\frac{f_{n}^{\prime}}{\sqrt{2^{n-6}(n-6) !}}, \\
g_{n}=\frac{g_{n}^{\prime}}{\sqrt{2^{n-4}(n-4) !}}, \quad h_{n}=\frac{h_{n}^{\prime}}{\sqrt{2^{n-2}(n-2) !}}, \\
t_{n}=\frac{t_{n}^{\prime}}{\sqrt{2^{n+2}(n+2) !}}, \quad u_{n}=\frac{u_{n}^{\prime}}{\sqrt{2^{n+4}(n+4) !}}, \\
v_{n}=\frac{v_{n}^{\prime}}{\sqrt{2^{n+6}(n+6) !}}, \quad w_{n}=\frac{w_{n}^{\prime}}{\sqrt{2^{n+8}(n+8) !}} .
\end{array}
$$

\section{References}

[1] N. P. Andion, A. P. C. Malbouisson and A. Mattos Neto, J. Phys. A34, 3735, (2001).

[2] G. Flores-Hidalgo, A. P. C. Malbouisson and Y. W. Milla, Phys. Rev. A65, 063414 (2002); physics/0111042. 
[3] G. Flores-Hidalgo and A.P.C. Malbouisson, Phys. Rev. A66, 042118 (2002); quant$\mathrm{ph} / 0205042$.

[4] R. Casana, G. Flores-Hidalgo and B. M. Pimentel, physics/0410063.

[5] R. G. Hulet, E. S. Hilfer, D. Kleppner, Phys. Rev. Lett. 55, 2137 (1985).

[6] W. Jhe, A. Anderson, E. A. Hinds, D. Meschede, L. Moi and S. Haroche, Phys. Rev. Lett. 58, 666 (1987).

[7] G. Flores-Hidalgo and A. P. C. Malbouisson, Phys. Lett. A311, 82 (2003); physics/0211123.

[8] W. Thirring and F. Schwabl, Ergeb. Exakt. Naturw. 36, 219 (1964).

[9] U. Weiss, Quantum dissipative systems, (World Scientific Publishing Co., Singapore, 1993).

[10] G. W. Ford, J. T. Lewis and R. F. O'Connell, J. Stat. Phys. 53, 39 (1988).

[11] G. Flores-Hidalgo and R. O. Ramos, Physica A326, 159 (2003); hep-th/0206022.

[12] S. Kim and G. Ordonez, Phys. Rev. A66, 012109 (2002).

[13] N. Polonsky, doctoral thesis, (Ecole Normale Supérieure, Paris, 1964).

[14] S. Haroche, doctoral thesis, (Ecole Normale Supérieure, Paris, 1964).

[15] C. Cohen-Tannoudji, J. Dupont-Roc and G. Grynberg, Atom-Photons Interactions (John Wiley \& Sons, Inc., New York, 1992).

[16] C. Cohen-Tannoudji, Atoms in Electromagnetic Fields, (World Scientific, Singapore, 1994).

[17] L. Skála, J. Čížek, J. Dvořák and V. Špirko, Phys. Rev. A53, 2009 (1996). 